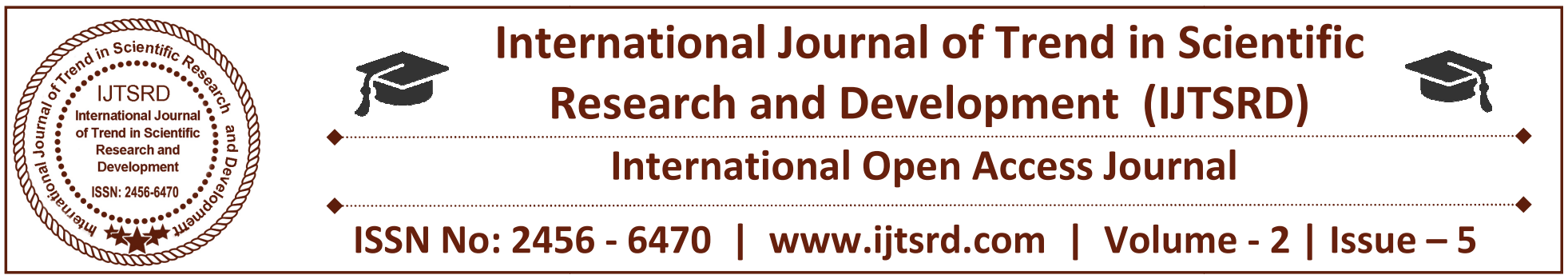

\title{
Common Fixed Point Theorems Using R-Weakly Commuting Mappings
}

\author{
Rashmi Rani \\ Assistant Professor, A. S. College for Women, \\ Khanna, Punjab, India
}

\begin{abstract}
In this paper, we prove a common fixed point theorem in modified intuitionistic fuzzy metric space by combining the ideas of point wise $\mathrm{R}$ - weak commutativity and reciprocal continuity of mappings satisfying contractive conditions. We also give example to prove validity of proved result.
\end{abstract}

Key words: Compatible maps; R-weakly commuting mappings; modified intuitionistic fuzzy metric space. AMS (2010) Subject Classification: 47H10, $54 \mathrm{H} 25$

\section{INTRODUCTION AND PRELIMINARIES:}

Recently, R. Saadati et. al [10] introduced the modified intuitionistic fuzzy metric space and proved some fixed point theorems for compatible and weakly compatible maps. The paper [10] is the inspiration of a large number of papers $[1-3,6-9,11]$ that employ the use of modified intuitionistic fuzzy metric space and its applications.

In this paper, we prove a common fixed point theorem in modified intuitionistic fuzzy metric space by combining the ideas of point wise $R$ - weak commutativity and reciprocal continuity of mappings satisfying contractive conditions. We also give example to prove validity of proved result.

Firstly, we recall the following notions that will be used in the sequel.

Lemma 1.1 [4]:

Consider the set $L^{*}$ and the operation $\leq_{L^{*}}$ defined by

$$
\begin{aligned}
& L^{*}=\left\{\left(x_{1}, x_{2}\right):\left(x_{1}, x_{2}\right) \in[0,1]^{2}, x_{1}+x_{2} \leq 1\right\} \\
& \left(x_{1}, x_{2}\right) \leq_{L^{*}}\left(y_{1}, y_{2}\right) \Leftrightarrow x_{1} \leq y_{1}, x_{2} \geq y_{2},
\end{aligned}
$$

For every $\left(x_{1}, x_{2}\right),\left(y_{1}, y_{2}\right)$ in $L^{*}$, Then $\left(L^{*}, \leq_{L^{*}}\right)$ is a complete lattice.

We denote its units by $0_{L^{*}}=(0,1)$ and $1_{L^{*}}=(1,0)$.

\section{Definition 1.1 [5]:}

A triangular norm ( $t$-norm) on $L^{*}$ is a mapping $\mathrm{F}$ : $\left(L^{*}\right)^{2} \rightarrow L^{*}$ satisfying the following conditions:

1. $\mathrm{F}\left(x, 1_{L^{*}}\right)=x$ for all $x$ in $L^{*}$,

2. $\mathrm{F}(x, y)=\mathrm{F}(y, x)$ for all $x, y$ in $L^{*}$,

3. $\mathrm{F}(x, \mathrm{~F}(\mathrm{y}, \mathrm{z}))=\mathrm{F} \quad(\mathrm{F} \quad(\mathrm{x}, \mathrm{y}), \mathrm{z})$ for all $x, y, z$ in $L^{*}$,

4. If for all $x, x^{\prime}, y, y^{\prime}$ in $L^{*}, x \leq_{L^{*}} x^{\prime}$ and $y \leq_{L^{*}} y^{\prime}$ implies $\mathrm{F}_{-}(x, y) \leq_{\mathrm{L}^{*}} \mathrm{~F} \quad\left(x^{\prime}, y^{\prime}\right)$.

\section{Definition 1.2 [4,5]:}

A continuous $t$-norm $\mathrm{F}$ on $L^{*}$ is called continuous $t-$ represent table if there exist a continuous $t$ - norm * and a continuous $t-$ co norm $\diamond$ on $[0,1]$ such that for all $x=\left(x_{1}, x_{2}\right), y=\left(y_{1}, y_{2}\right) \in L^{*}[0,1]^{2}, \quad \mathrm{~F}(x, y)=$ $\left(x_{1}^{*} y_{1}, x_{2} \diamond y_{2}\right)$.

\section{Definition 1.3 [10]:}

Let $M, N$ are fuzzy sets from $X^{2} \times(0,+\infty) \rightarrow[0,1]$ such that $M(x, y, t)+N(x, y, t) \leq 1$ for all $x, y$ in $X$ and $t>0$. The 3 - tuple $\left(X, \zeta_{M, N}, \mathrm{~F}\right)$ is said to be a modified intuitionistic fuzzy metric space if $X$ is an arbitrary non empty set, $\mathrm{F}$ is a continuous $t$ represent table and $\zeta_{M, N}$ is a mapping $X^{2} \times(0,+\infty) \rightarrow L^{*} \quad$ satisfying the following conditions for every $x, y$ in $X$ and $t, s>0$ :
(a) $\zeta_{M, N}(x, y, t)>_{\mathrm{L}^{*}} 0_{\mathrm{L}^{*}}$;
(b) $\zeta_{M, N}(x, y, t)=1_{L^{*}}$ if $x=y$;
(c) $\zeta_{M, N}(x, y, t)=\zeta_{M, N}(y, x, t)$; 
(d) $\zeta_{M, N}(x, y, t+s) \geq_{L^{*}} \mathrm{~F}\left(\zeta_{M, N}(x, z, t), \zeta_{M, N}(z, y\right.$, $s))$

(e) $\zeta_{M, N}(x, y,):.(0,+\infty) \rightarrow L^{*}$ is continuous.

In this case, $\zeta_{M, N}$ is called a modified intuitionistic fuzzy metric. Here,

$\zeta_{M, N}(X, y, t)=(M(x, y, t), N(x, y, t))$.

\section{Remark1.1. [11]:}

In a modified intuitionistic fuzzy metric space $(X$, $\left.\zeta_{M, N}, \mathrm{~F}\right), M(x, y,$.$) is non decreasing and N(x, y,$. is non- increasing for all $x, y$ in $X$. Hence $\zeta_{M, N}(x, y$, $t$ ) is non- decreasing with respect to $t$ for all $x, y$ in $X$.

\section{Definition 1.4 [10]:}

A sequence $\left\{x_{n}\right\}$ in a modified intuitionistic fuzzy metric space

$\left(X, \zeta_{M, N}, \mathrm{~F}\right)$ is called a Cauchy sequence if for each $\varepsilon>0$ and $t>0$, there exists $n_{0} \in \square$ such that $\zeta_{M, N}\left(x_{n}, x_{m}, t\right)>_{L^{*}}(1-\varepsilon, \varepsilon)$ for each $n, m \geq n_{0}$ and for all $t$.

\section{Definition 1.5 [10]:}

A sequence $\left\{x_{n}\right\}$ in a modified intuitionistic fuzzy metric space

$\left(X, \zeta_{M, N}, \mathrm{~F}\right)$ is said to be convergent to $x$ in $X$, denoted by $x_{n} \rightarrow x$ if

$\lim _{n \rightarrow \infty} \zeta_{M, N}\left(x_{n}, x, t\right)=1_{L^{*}}$ for all $t$.

\section{Definition 1.6 [10]:}

A modified intuitionistic fuzzy metric space $\left(X, \zeta_{M, N}\right.$, $F)$ is said to be complete if every Cauchy sequence is converges to a point of it.

\section{Definition $1.7[10,11]$}

A pair of self mappings $(f, g)$ of modified intuitionistic fuzzy metric space $\left(X, \zeta_{M, N}, \mathrm{~F}\right)$ is said to be compatible if $\lim _{n \rightarrow \infty} \zeta_{M, N}\left(f g x_{n}, g f x_{n}, t\right)=1_{L^{*}}$ whenever $\left\{x_{n}\right\}$ is a sequence in $X$ such that $\lim _{n \rightarrow \infty} f\left(x_{n}\right)=\lim _{n \rightarrow \infty} g\left(x_{n}\right)=z$ for some $z$ in $X$.

\section{Definition 1.8 [11]}

Two self-mappings $f$ and $g$ are called non-compatible if there exists at least one sequence $\left\{x_{n}\right\}$ such that $\lim _{n \rightarrow \infty} f\left(x_{n}\right)=\lim _{n \rightarrow \infty} g\left(x_{n}\right)=z$ for some $z$ in $X$ but either $\lim _{n \rightarrow \infty} \zeta_{M, N}\left(f g x_{n}, g f x_{n}, t\right) \neq 1_{L^{*}}$ or the limit does not exist for all $\mathrm{z}$ in $\mathrm{X}$.

\section{Definition 1.9 [11]:}

A pair of self mappings $(f, g)$ of a modified intuitionistic fuzzy metric space $\left(X, \zeta_{M, N}, \mathrm{~F}\right)$ is said to be $R$-weakly commuting at a point $x$ in $X$ if $\zeta_{M, N}\left(f g x_{n}, g f x_{n}, t\right) \geq_{L^{*}} \zeta_{M, N}\left(f x_{n}, g x_{n}, t / R\right)$ for some $R>0$.

\section{Definition 1.10[11]:}

The two self-maps $f$ and $g$ of a modified intuitionistic fuzzy metric space $\left(X, \zeta_{M, N}, \mathrm{~F}\right)$ are called point wise $R$-weakly commuting on $X$ if given $x$ in $X$ there exists $R \rightarrow 0 \quad 0 \quad$ such that $\zeta_{M, N}(f g x, g f x, t) \geq_{L^{*}} \zeta_{M, N}(f x, g x, t / R)$.

\section{Lemmas:}

The proof of our result is based upon the following Lemmas:

\section{Lemma2.1[8].}

Let $\left(X, \zeta_{M, N}, \mathrm{~T}\right)$ be modified intuitionistic fuzzy metric space and for all $x, y \in X, t>0$ and if for a number $k \in(0,1)$,

$\zeta_{M, N}(x, y, k t) \geq_{L^{*}} \zeta_{M, N}(x, y, t)$.

Then $x=y$.

Lemma2.2[8].

Let $\left(X, \zeta_{M, N}, \mathrm{~T}\right)$ be modified intuitionistic fuzzy metric space and $\left\{y_{n}\right\}$ be a sequence in $X$. If there exists a number $k \in(0,1)$ such that:

$\zeta_{M, N}\left(y_{n}, y_{n+1}, k t\right) \geq_{L^{*}} \zeta_{M, N}\left(y_{n-1}, y_{n}, t\right)$

For all $t>0$ and $n=1,2,3, \ldots \ldots$.

Then $\left\{y_{n}\right\}$ is a Cauchy sequence in $X$.

\section{Main Results:}

\section{Lemma 3.1:}

Let $\left(X, \zeta_{M, N}, T\right)$ be a modified intuitionistic fuzzy metric space and let $(A, S)$ and $(B, T)$ be pairs of self mappings on $X$ satisfying

(3.1) $A(X) \subseteq T(X), B(X) \subseteq S(X)$,

(3.2) there exists a constant $k \in(0,1)$ such that 
$\zeta_{M, N}(A x, B y, k t) \geq L^{*} \min \left\{\zeta_{M, N}(T y, B y, t), \quad \zeta_{M, N}\right.$ $\left.(S x, A x, t), \zeta_{M, N}(S x, B y, \alpha t)\right\}$

For all $x, y \in X, t>0$ and $\alpha \in(0,2)$. Then the continuity of one of the mappings in compatible pair $(A, S)$ or $(B, T)$ on $\left(X, \zeta_{M, N}, T\right)$ implies their reciprocal continuity.

\section{Proof:}

Proof of this result easily follows condition (3.2) and definition of compatible maps.

\section{Theorem 3.1:}

Let $\left(X, \zeta_{M, N}, \mathrm{~T}\right)$ be a complete modified intuitionistic fuzzy metric space. Further, let $(A, S)$ and $(B, T)$ be point wise $R$ - weakly commuting pairs of self mappings of $X$ satisfying (3.1), (3.2). If one of the mappings in compatible pair $(A, S)$ or $(B, T)$ is continuous, then $A, B, S$ and $T$ have a unique common fixed point in $X$,

\section{Proof:}

Let $x_{0} \in X$. By (3.1), we define the sequences $\left\{x_{n}\right\}$ and $\left\{y_{n}\right\}$ in $X$ such that for all $n=0,1,2 \ldots$

$y_{2 n}=A x_{2 n}=T x_{2 n+1}, y_{2 n+1}=B x_{2 n+1}=S x_{2 n+2}$.

We show that $\left\{y_{n}\right\}$ is a Cauchy sequence in $X$. By (3.2) take $\alpha=1-\beta, \beta \in(0,1)$, we have

$\zeta_{M, N}\left(y_{2 n+1}, y_{2 n+2}, k t\right)=\zeta_{M, N}\left(B x_{2 n+1}, A x_{2 n+2}, k t\right)=\zeta_{M, N}\left(A x_{2 n+2}, B x_{2 n+1}, k t\right)$

$\geq_{L^{2}} \min \left\{\zeta_{M, N}\left(T x_{2 n+1}, B x_{2 n+1}, t\right), \zeta_{M, N}\left(S x_{2 n+2}, A x_{2 n+2}, t\right), \zeta_{M, N}\left(S x_{2 n+2}, B x_{2 n+1},(1-\beta) t\right),\right\}$

$=\min \left\{\zeta_{M, N}\left(y_{2 n}, y_{2 n+1}, t\right), \zeta_{M, N}\left(y_{2 n+1}, y_{2 n+2}, t\right), \zeta_{M, N}^{a t}\left(y_{2 n+1}, y_{2 n+1},(1-\beta) t\right\}\right.$

$=\min \left\{\zeta_{M, N}\left(y_{2 n}, y_{2 n+1}, t\right), \zeta_{M, N}\left(y_{2 n+1}, y_{2 n+2}, t\right), 1_{L^{2}}\right\}$

$\geq_{L^{*}} \min \left\{\zeta_{M, N}\left(y_{2 n}, y_{2 n+1}, t\right), \zeta_{M, N}\left(y_{2 n+1}, y_{2 n+2}, t\right), \zeta_{M, N}\left(y_{2 n+1}, y_{2 n+2}, \beta t\right)\right\}$

Taking $\beta \rightarrow 1$, we have

$\zeta_{M, N}\left(y_{2 n+1}, y_{2 n+2}, k t\right) \geq_{L^{\prime}} \min \left\{\zeta_{M, N}\left(y_{2 n}, y_{2 n+1}, t\right), \zeta_{M, N}\left(y_{2 n+1}, y_{2 n+2}, t\right), \zeta_{M, N}\left(y_{2 n+1}, y_{2 n+2}, t\right)\right\}$

$\zeta_{M, N}\left(y_{2 n+1}, y_{2 n+2}, k t\right) \geq_{L^{*}} \min \left\{\zeta_{M, N}\left(y_{2 n}, y_{2 n+1}, t\right), \zeta_{M, N}\left(y_{2 n+1}, y_{2 n+2}, t\right)\right\}$

$\zeta_{M, N}\left(y_{2 n+1}, y_{2 n+2}, k t\right) \geq_{L^{*}} \zeta_{M, N}\left(y_{2 n}, y_{2 n+1}, t\right)$

\section{Similarly}

$\zeta_{M, N}\left(y_{2 n+2}, y_{2 n+3}, k t\right) \geq_{L^{*}} \zeta_{M, N}\left(y_{2 n+1}, y_{2 n+2}, t\right)$

Therefore, for any $n$ and $t$, we have

$\zeta_{M, N}\left(y_{n}, y_{n+1}, k t\right) \geq_{L^{*}} \zeta_{M, N}\left(y_{n-1}, y_{n}, t\right)$

Hence, by Lemma 2.2, $\left\{y_{n}\right\}$ is a Cauchy sequence in $X$. Since $X$ is complete, $\left\{y_{n}\right\}$ converges to $z$ in $X$. Its subsequences $\left\{A x_{2 n}\right\},\left\{T x_{2 n+1}\right\},\left\{B x_{2 n+1}\right\}$ and $\left\{S x_{2 n+2}\right\}$ also converges to $z$.

Now, suppose that $(A, S)$ is a compatible pair and $S$ is continuous. Then by Lemma 2.1, $A$ and $\mathrm{S}$ are reciprocally continuous, then $S A x_{n} \rightarrow S z, A S x_{n} \rightarrow A z$ as $n \rightarrow \infty$. As, $\quad(A, S)$ is a compatible pair. This implies

$\lim _{n \rightarrow \infty} \zeta_{M, N}\left(A S x_{n}, S A x_{n}, t\right)=1_{L^{*}}$

$\zeta_{M, N}(A z, S z, t)=1_{L^{*}}$.

Hence, $A z=S z$.

Since $A(X) \subseteq T(X)$, there exists a point $p$ in $X$ such that $A z=T p=S z$.

By (3.2), take $\alpha=1$, 
$\zeta_{M, N}(A z, B p, k t) \geq_{L^{*}} \min \left\{\zeta_{M, N}(T p, B p, t), \zeta_{M, N}(S z, A z, t), \zeta_{M, N}(S z, B p, t),\right\}$

$\zeta_{M, N}(A z, B p, k t) \geq_{L^{*}} \min \left\{\zeta_{M, N}(A z, B p, t), \zeta_{M, N}(A z, A z, t), \zeta_{M, N}(A z, B p, t),\right\}$

$\zeta_{M, N}(A z, B p, k t) \geq_{L^{*}} \zeta_{M, N}(A z, B p, t)$

Thus, by Lemma 2.1, we have $A z=B p$.

Thus, $A z=B p=S z=T p$.

Since, $A$ and $S$ are point wise $R$ - weakly commuting mappings, there exists $R>0$, such that

$$
\zeta_{M, N}(A S z, S A z, t) \geq_{L^{*}} \zeta_{M, N}(A z, S z, t / R)=1_{L^{*}}
$$

Therefore, $A S z=S A z$ and $A A z=A S z=S A z=S S z$.

Similarly, $B$ and $T$ are point wise $R$-weakly commuting mappings, we have $B B p=B T p=T B p=T T p$.

Again by (3.2), take $\alpha=1$,

$$
\begin{aligned}
& \zeta_{M, N}(A A z, B p, k t) \geq_{L^{*}} \min \left\{\zeta_{M, N}(T p, B p, t), \zeta_{M, N}(S A z, A A z, t), \zeta_{M, N}(S A z, B p, t),\right\} \\
& \zeta_{M, N}(A A z, A z, k t) \geq_{L^{*}} \min \left\{\zeta_{M, N}(T p, T p, t), \zeta_{M, N}(A A z, A A z, t), \zeta_{M, N}(A A z, A z, t),\right\} \\
& \zeta_{M, N}(A A z, A z, k t) \geq_{L^{*}} \zeta_{M, N}(A A z, A z, t) \text { ternational Journal }
\end{aligned}
$$

By Lemma 2.1, we have $A A z=A z=S A z$. Hence $A z$ is common fixed point of $A$ and $S$. Similarly by (3.2), $B p$ $=A z$ is a common fixed point of $B$ and $T$. Hence, $A z$ is a common fixed point of $A, B, S$ and $T$.

\section{For Uniqueness:}

We can easily prove uniqueness by using (3.2).

\section{Corollary 3.1:}

Let $\left(X, \zeta_{M, N} \mathrm{~T}\right)$ be a complete modified intuitionistic fuzzy metric space. Further, let $A$ and $B$ are reciprocally continuous mappings on $X$ satisfying

(3.3) $\zeta_{M, N}(A x, B y, k t) \geq_{L^{*}} \min \left\{\zeta_{M, N}(y, B y, t), \zeta_{M, N}(x, A x, t), \zeta_{M, N}(x, B y, \alpha t)\right\}$

For all $x, y \in X, t>0$ and $\alpha \in(0,2)$ then pair $A$ and $B$ has a unique common fixed point.

\section{Example 2.1:}

Let $X=[0,20]$ and for each $t>0$, define $\zeta_{M, N}(x, y, t)=\left(\frac{t}{t+|x-y|}, \frac{|x-y|}{t+|x-y|}\right)$. Then $\left(X, \zeta_{M, N}, \mathrm{~T} \quad\right)$ is complete modified intuitionistic fuzzy metric space. Let $A, B, S$ and $T$ be self mappings of $\mathrm{X}$ defined as

$A(2)=2, A u=3$ if $\mathrm{u}>0$,

$B(\mathrm{u})=2$ if $u=2$ or $\mathrm{u}>6, B u=6$ if $0<u \leq 6$,

$S(2)=2, S(u)=6$ if $u>0$,

$T(2)=2, T(u)=12$ if $0<u \leq 6, T(u)=u-3$ if $u>6$.

Then $A, B, S$ and $T$ satisfy all the conditions of above theorem with $k \in(0,1)$ and have a unique common fixed point $u=2$. 


\section{References}

1. H. Adibi, Y. J. Cho, D. O'Regan and R. Saadati, Common fixed point theorems in $L$-fuzzy metric spaces, Appl. Math. Comput., 182(1)(2006), 820828.

2. A. T. Atanassov, Intuitionistic fuzzy sets, Fuzzy Sets and Systems, 20 (1986), 87-96.

3. D. Coker, An introduction to Intuitionistic Fuzzy topological spaces, Fuzzy Sets and System, 88 (1997), 81- 89.

4. G. Deschrijver and E. E. Kerre, on the relationship between some extensions of fuzzy set theory. Fuzzy Sets and Systems, 133 (2003), 227-235.

5. G. Deschrijver, C. Cornelis and E. E. Kerre, on the representation of intuitionistic fuzzy $t$-norm and $t$-co norm, IEEE Trans. Fuzzy System, 12 (2004), 45-61.

6. I. Kramosil and J. Michalek, Fuzzy metric and statistical metric spaces, Kybernetica 11 (1975), 326-334.

7. S. Manro, S. Kumar, S. S. Bhatia and K. Tas, Common fixed point theorems in modified intuitionistic fuzzy metric spaces, Journal of Applied Mathematics, Volume 2013, Article ID 189321, 13 pages.

8. S. Manro and Sumitra, Common new fixed point theorem in modified intuitionistic fuzzy metric spaces using implicit relation, Applied Mathematics, 4(2013), 27-31.

9. R. Saadati and J. H. Park, on the intuitionistic fuzzy topological spaces, Chaos Solitons \& Fractals 27(2006), 331-344.

10. R. Saadati, S. Sedghi and N. Shob he, Modified Intuitionistic fuzzy metric spaces and some fixed point theorems. Chaos, Soli tons \& Fractals 38 (2008), 36-47.

11. R. Saadati, S. Sedghi and H Zhou, A common fixed point theorem for $\psi$ weakly commuting maps in L- fuzzy metric spaces, Iranian Journal of Fuzzy Systems, 5(1)(2008),47-53. 